\title{
Large area silicon-air-silicon DBRs for infrared filter applications
}

\author{
Jorge R. Silva, Hemendra Kala, Dhirendra Kumar Tripathi, Member, IEEE, \\ K. K. M. B. Dilusha Silva, Member, IEEE, Mariusz Martyniuk, Adrian Keating, Gino Putrino, \\ Lorenzo Faraone, Fellow, IEEE,
}

\begin{abstract}
This paper presents the design, fabrication, and optical characterization of silicon-based thin film Fabry-Pérot filters for spectroscopic sensing applications at short-wave infrared (SWIR: 1.5-2.5 $\mu \mathrm{m}$ ) and mid-wave infrared (MWIR: 3-5 $\mu \mathrm{m}$ ) wavelengths. Filter performance is enhanced using distributed Bragg reflectors composed of silicon and air-gap layers for enhanced refractive index contrast. A peak-topeak surface variation of less than $20 \mathrm{~nm}$ in the fabricated micromachined structures was achieved across a large spatial area of $1 \mathrm{~mm} \times 1 \mathrm{~mm}$. Spectral measurements on released Fabry-Pérot filters show excellent agreement with optical simulations. The fabricated Fabry-Pérot filters demonstrate peak transmittance values greater than $50 \%$ across all spectral ranges, with measured full width at half maximum values in the range of $50 \mathrm{~nm}$ rendering them suitable for use in spectral sensing and imaging in the SWIR and MWIR wavelength ranges.
\end{abstract}

Index Terms-Fabry-Perot interferometers, Spectroscopy, Silicon, Optical device fabrication.

\section{INTRODUCTION}

Spectroscopy across both the short-wave infrared (SWIR: 1.5-2.5 $\mu \mathrm{m}$ ) and mid-wave infrared (MWIR: 3-5 $\mu \mathrm{m}$ ) ranges takes advantage of important absorption peaks and spectral signatures of materials to obtain high precision measurements for industrial applications such as gas leak detection [1], wildfire detection [2], and mineral exploration. Within the field of precision agriculture, these systems are used for crop health [3], [4], [5], plant stress [3], [4], [5], soil moisture [3], [4], and soil organic matter analysis [3], [4], [6]. While spectral sensing is relatively simple in the SWIR band due to the availability of room-temperature detection, spectral features of detected analytes in the SWIR band tend to have broad overtones and mixing terms, requiring high-power computing, and statistical or machine learning methods to extract quantitative information regarding the sample composition [7]. On the other hand, fundamental resonances in the MWIR are easier to analyse, although cooled detectors are needed.

Microelectromechanical systems (MEMS) technology has paved the way for producing low cost, highly portable, robust spectroscopic systems with high spectral and spatial resolution. These microspectrometers can be created by combining MEMS tunable filters with existing low cost detectors or focal plane arrays (FPAs). Such systems can enable the widespread application of spectroscopy for agriculture and other industries

Authors are with the School of Electrical, Electronic and Computer Engineering, University of Western Australia, Perth, WA 6009, Australia. Contact e-mail: jorge.silvacastillo@ research.uwa.edu.au,dilusha.silva@uwa.edu.au. where the size, weight and power (SWaP) requirements are very strict [3], [4], [5], [6]. For example, due to their low SWaP, MEMS spectroscopic systems can be deployed easily on small drones, enabling low cost aerial spectroscopy for onfarm applications [8], [9]. These systems can overcome the SWaP limitations of typical bench top spectroscopic systems which, while offering high resolution and a wide wavelength range, tend to be bulky, expensive and fragile. MEMS based spectrometers for the SWIR and MWIR wavelength ranges have been the subject of ongoing research by multiple groups [10], [11], [12], [13], [14], [15]. The most common approach for their realisation is based on tunable Fabry-Pérot (FP) filters consisting of two distributed Bragg reflectors (DBR) separated by an air gap that can be adjusted electrically to control the central wavelength of the FP filter. As metal mirrors tend to be lossy, dielectric DBRs are generally used to provide high reflectivity with low loss. A 3-layer DBR consists of two high refractive index dielectric layers separated by a low refractive index layer, with each layer being a quarter wavelength in optical thickness. A higher refractive index contrast between the high and low index mirror layers yields higher reflectivity and extends the free spectral range [16]. By increasing the reflectivity of the DBRs, a narrower full width half maximum (FWHM) and an improved out-of-band rejection of the FP filter can be achieved. In addition, the mirror reflectivity plays a crucial role in determining the wavelength selectivity as well as the signal-to-noise ratio (SNR) of the spectrometer. The choice of high and low index materials in the DBR determines not only the optical performance of the FP filter, but also the mechanical behaviour and fabrication complexity of the entire MEMS structure. In this work, silicon is used as the high refractive index material as it offers two key advantages. Firstly, the optical properties of silicon allow operation of the DBRs and FP filters to span both the SWIR and the MWIR optical wavelength region. Secondly, since silicon is an established MEMS structural material, the fabrication process is industry-compatible and simplified by way of eliminating the need for a separate structural material. In the top suspended $\mathrm{DBR}$, this work uses air as the low refractive index layer since it provides the lowest loss, lowest refractive index natural layer possible, thus providing the ultimate refractive index contrast for the DBR stack in comparison to any other low refractive index material. This paper presents the structural design, optical and mechanical simulations, fabrication process and optical characteristics of FP filters based on suspended siliconair-silicon DBRs targeting their application for high spectral 
resolution IR spectrometry for deployment in platforms with very strict $\mathrm{SWaP}$ requirements.

\section{StRuctural DESIGN OF DBRS AND FP FILTERS}

A cross-sectioned view of the proposed Si-air-Si FP filter is shown in Figure 1 (a), and its plain view shown in Figure 1 (b), with each layer thickness used for simulation and fabrication of both SWIR and MWIR DBRs and FP filters being shown in Table I. The structure uses a 4-layer solid DBR as the bottom mirror and a Si-air-Si suspended DBR as the top mirror, with each layer being of equivalent quarter-wave optical thickness. The chosen design has three main advantages, namely: (1) a 4-layer bottom-mirror attached to the substrate does not require stress balancing, reduces lithography and process complexity by requiring only two air-cavities to be released in the entire filter structure, and allows the bottom mirror to closely match the reflectivity of the suspended Si-air-Si DBR for best performance of the FP filter; (2) stress-balancing is simpler in a $\mathrm{Si}$-air-Si suspended DBR in comparison to a suspended 4-layer solid DBR structure containing different materials; and (3) since air has the lowest loss, lowest refractive index possible, this improves the optical performance of the top DBR compared to that of a 3-layer DBR with a solid low index layer such as silicon oxide.

TABLE I

THICKNESS OF MIRROR LAYERS USED FOR BOTH SIMULATED AND FABRICATED FILTERS.

\begin{tabular}{|c||c|c|}
\hline $\begin{array}{c}\text { Wavelength } \\
\text { Range } \\
\text { (nm) }\end{array}$ & $\begin{array}{c}\text { Layer } \\
\text { Material }\end{array}$ & $\begin{array}{c}\text { Layer } \\
\text { Thickness } \\
\text { (nm) }\end{array}$ \\
\hline \hline \multirow{2}{*}{ SWIR } & Silicon & 145 \\
$(1500-2500)$ & $\begin{array}{c}\text { Silicon Oxide } \\
\text { Air spacer } \\
\text { (Prolift100-16) }\end{array}$ & 360 \\
\hline \multirow{2}{*}{ MWIR } & $\begin{array}{c}\text { Silicon } \\
\text { Silicon Oxide } \\
\text { Air spacer } \\
\text { (3000-5000) }\end{array}$ & 720 \\
& $\begin{array}{c}\text { (Prolift100-16) } \\
\text { (2000 }\end{array}$ \\
\hline
\end{tabular}

\section{OPTICAL AND MECHANICAL MODELLING OF THE FILTERS}

The optical modelling of ideal FP filters incorporating a Si-air-Si top DBR was carried out using the basic optical matrix method [16] in order to estimate the best-case optical spectrum transmittance and reflectance of both the DBRs and FP filters. The model was extended to accommodate for surface deformation in 2-dimensions by representing the FP filter with imperfections as an array of parallel ideal FP sub-filters with different air gaps. Figure 2 depicts this process in 2-dimensions, where the continuous surface profile is approximated by an array of locally-flat regions. The fractional contribution of each ideal FP sub-filter to the overall transmission of the non-ideal FP filter is in proportion to the fraction of incident light that passes over the area of each FP sub-filter, according to the beam intensity distribution over the optical area of the FP filter [17, pp 23-31]. These assumptions only hold true when the standard deviation of the imperfections of the layers are very small compared to the lateral dimensions in the optical area of the FP filter. The transmission of the imperfect FP filter is obtained by averaging the spectral transmission of all the sub-filters. This model extension assumes that the beam intensity is uniformly distributed over the filter area. The extended modelling is performed here on a surface profile that is either radially symmetric, or has tilt only in one dimension. As such, these two geometries allow a 1D simulation to produce a prediction equivalent to $2 \mathrm{D}$.

This extended model was used to simulate the effect of unwanted tilt and concave bowing, which arise from sacrificial layer non-uniformities or residual stress, on the spectral transmittance and reflectance of DBRs and FP filters [18]. For these simulations it was assumed that the suspended Si-air$\mathrm{Si}$ DBR layers $\left(\mathrm{Si}^{I}\right.$ and $\mathrm{Si}^{I I}$ layers in Figure 1 (a)) remain parallel by deflecting the same amount and their relative thicknesses stay the same during that deflection. We define membrane concave bowing and tilt as the maximum deviation in height of a layer from its ideally flat position. This work required an investigation of flatness requirements for high performance FP filters. Optical modelling was used to study the impact on the optical transmittance of unwanted upward and downward concave bowing in the suspended layers, as detailed in Figure 3. A 2-Dimensional SWIR filter was used for these simulations using a nominal air cavity thickness of $1240 \mathrm{~nm}$ for the ideal filter (suspended Si layer perfectly flat and parallel to the substrate), with the thickness of each layer as described in Table I. The suspended layers are assumed to be constrained at the edges of the optical area, at a thickness of $1240 \mathrm{~nm}$, and the centre of the membrane is at the maximum upward or downward concave bowing displacement, as illustrated in Figure 3 (a). As upward concave bowing increases, the peak transmittance position undergoes a red shift because the mean value of the main air cavity thickness is greater than when the top DBR is flat, where as when downward concave bowing increases the peak transmittance position undergoes a blue shift because the mean value of the main air cavity thickness is less than that when the suspended DBR is flat. Furthermore, an increase in either upward or downward concave bowing causes the peak transmittance to be significantly reduced and the optical pass-band of the filter to broaden significantly [19]. Thus, any bowing in the suspended films needs to be minimized to less than $20 \mathrm{~nm}$. Compressive residual stress is considered the principle cause of edge-to-edge bowing in suspended thin films [20], which can be eliminated by depositing thin films with a slight residual tensile stress.

To investigate how much residual tensile stress is needed to maintain the thin suspended Si layers flat within $20 \mathrm{~nm}$, finite element modelling (FEM) of a suspended Si layer was undertaken using Coventorware 10.2 FEM suite [21] using the structure shown in Figure 4 (a). For this modelling, the Young's modulus and Poisson ratio were chosen as $130 \mathrm{GPa}$ and 0.27 , respectively. These values are typical for amorphous Si thin films deposited by the Sentech SI500D inductively coupled plasma chemical vapour deposition (ICPCVD) reactor used for device fabrication in this work [22]. Figure 4 


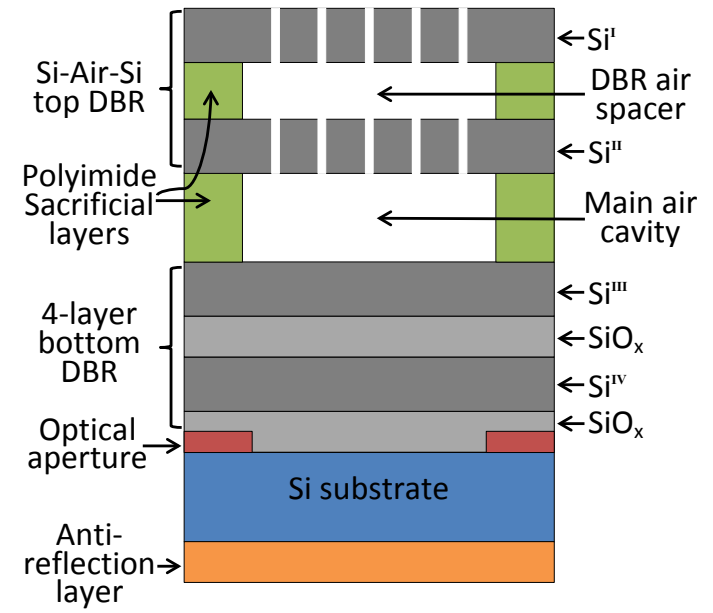

(a)

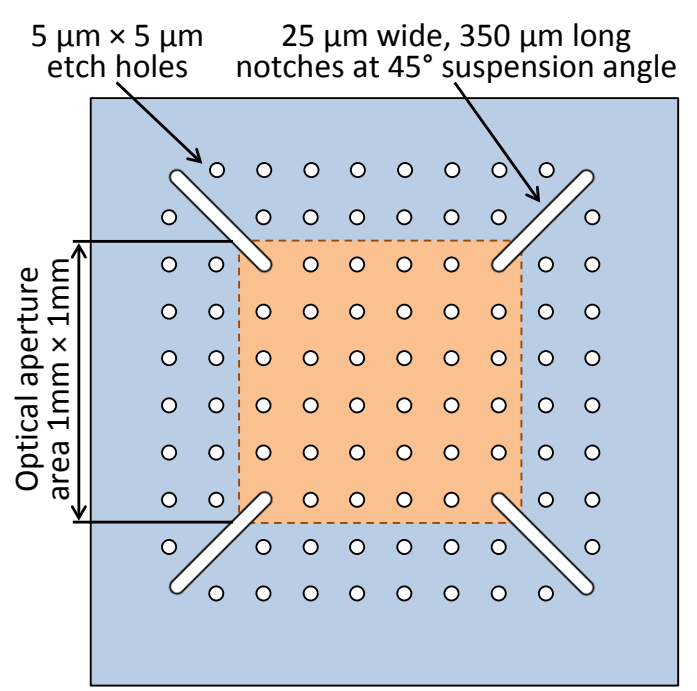

(b)

Fig. 1. Structure of the proposed Fabry-Pérot filter. (a) Cross-sectional view, and (b) a magnified top view depicting array of etch holes (dimensions not to scale).

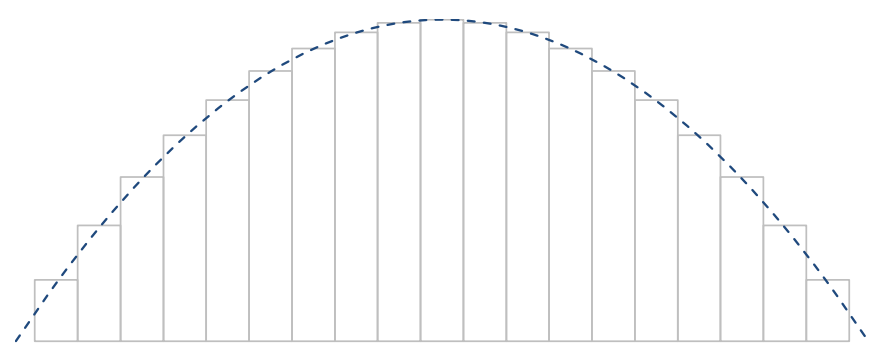

Fig. 2. Surface profile of a filter having a bowed top-membrane (dashed line), and representation of bowed filter as an array of locally-flat (bars) FP sub-filters with different air gaps.

shows membrane downward concave bowing as predicted by the model, for a $1 \mathrm{~mm}^{2}$ one-quarter wave thick suspended amorphous Si thin layer (thickness of $290 \mathrm{~nm}$ ). The initial downward concave bowing of the suspended layer is due to anchor effects and the different coefficients of thermal expansion between the silicon substrate wafer and the ICPCVD deposited $\mathrm{Si}$ of $2.6 \times 10^{-6}{ }^{\circ} \mathrm{C}^{-1}$ and $4.4 \times 10^{-6}{ }^{\circ} \mathrm{C}^{-1}$, respectively. We can observe from Figure 4 that as residual tensile stress increases beyond $75 \mathrm{MPa}$, the edge to edge concave bowing of the suspended thin film reduces to less than $10 \mathrm{~nm}$, and at $200 \mathrm{MPa}$ the concave bowing reduces to less than $5 \mathrm{~nm}$. However, it should be noted that excessive tensile stress can also lead to cracking/fracture of the suspended membrane [20]. Hence, in this work, the $\mathrm{Si}$ deposition recipe was optimised to yield suspended amorphous Si films with a residual tensile stress of 70-80 MPa.

Sacrificial layer thickness variations across the wafer may result in one edge of the membrane being higher or lower than the opposite edge, causing unwanted tilt which also degrades optical performance of DBRs and filters. To better understand the constraints imposed by this effect, a modelling study was carried out on the impact of suspended-layer downward tilt on the spectral transmittance and reflectance of the DBRs and FP filters. Initially, reflectance simulations of Si-air-Si DBRs on a low refractive index substrate of $n=1$ were undertaken assuming that the bottom suspended $\mathrm{Si}^{I I}$ layer is flat relative to the substrate, and the top suspended $\mathrm{Si}^{I}$ layer is downward tilted relative to the ideally flat position. The DBR air spacer nominal thickness is 500nm for SWIR DBRs and $1000 \mathrm{~nm}$ for MWIR DBRs. All other layer thickness are presented in Table I. DBR simulations using the extended optical matrix method were used to study the effect of tilt, assuming that one edge of the DBR is constrained at the ideal air spacer thickness, and the opposite edge decreases as much as the air spacer variation as illustrated in Figure 5 (a). The results of these simulations are plotted in Figure 5 (c) and (d), showing spectral reflectance under different tilt conditions, for SWIR and MWIR DBRs, respectively. In addition, reflectance simulations of ideal 4-layer $\mathrm{Si} / \mathrm{SiOx} / \mathrm{Si} / \mathrm{SiOx}$ solid DBRs on a high refractive index substrate of $n=3.4$ for the SWIR and MWIR ranges are also plotted in Figure 5 in order to compare their performance to that of Si-air-Si DBRs. It can be seen from Figure 5 that $\mathrm{Si}$-air-Si DBRs are better suited for high performance FP filters for both SWIR and MWIR spectral ranges since they exhibit high reflectivity over a wider spectral range as compared to 4-layer solid DBRs. Furthermore, the peak reflectivity of the SWIR and MWIR DBRs is essentially unaffected by tilt in the suspended Si thin film, although there is a blue-shift in the peak reflectance position with increased downward tilting of the top suspended $\mathrm{Si}^{I}$ layer because the air spacer mean value thickness is less compared to that when the $\mathrm{Si}^{I}$ layer is flat and parallel to both the substrate and $\mathrm{Si}^{I I}$ layer.

This Si-air-Si DBR forms the top suspended mirror of the proposed FP filter. The effect of tilt of the suspended Siair-Si DBRs relative to the substrate (that is, the DBR air spacer thickness is uniform, see Figure 6), on the spectral transmittance of the filter is estimated by using the extended optical matrix method discussed previously. Two-dimensional 


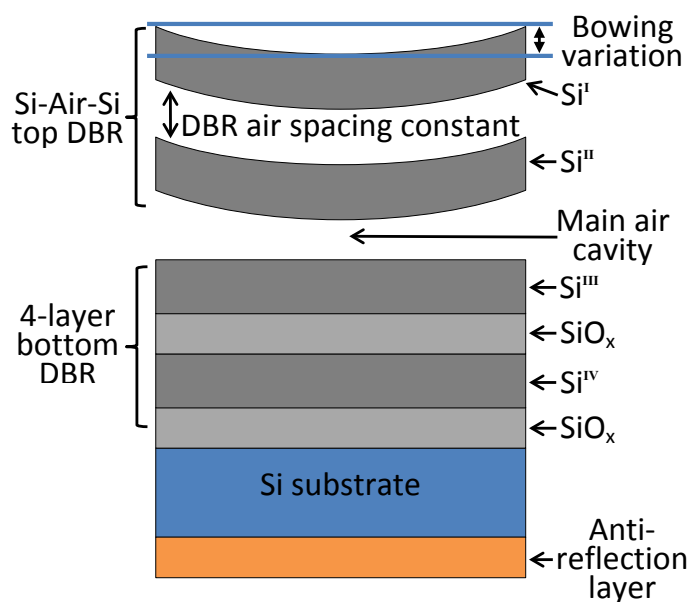

(a)

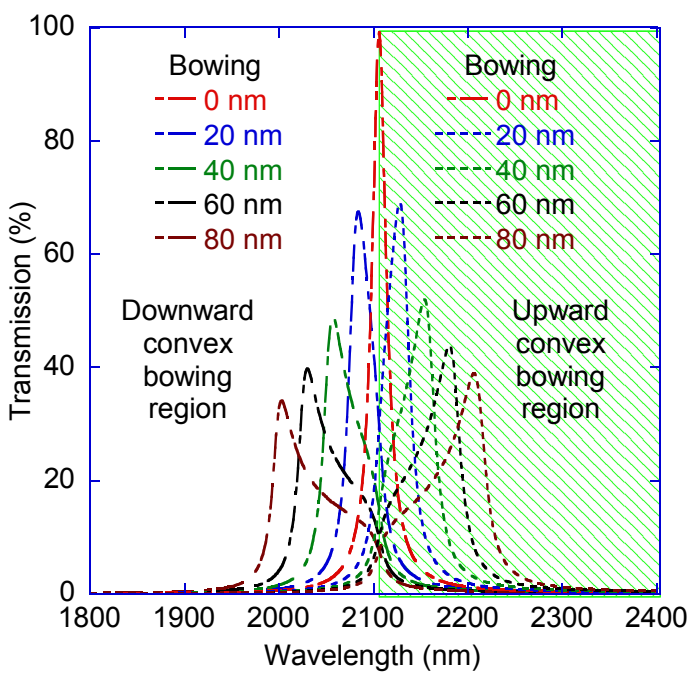

(b)

Fig. 3. Simulated spectral transmittance of a SWIR FP filter, using the structure proposed in Figure 1, as a function of wavelength for upward and downward concave bowing present in the $\mathrm{Si}^{I}$ and $\mathrm{Si}^{I I}$ layers (quarter wave optical thickness kept constant): (a) Cross section of the simulated filter displaying downward concave bowing and (b) Simulated spectral transmittance. Membrane concave bowing is defined as the maximum deviation in height of a layer from its ideally flat position.

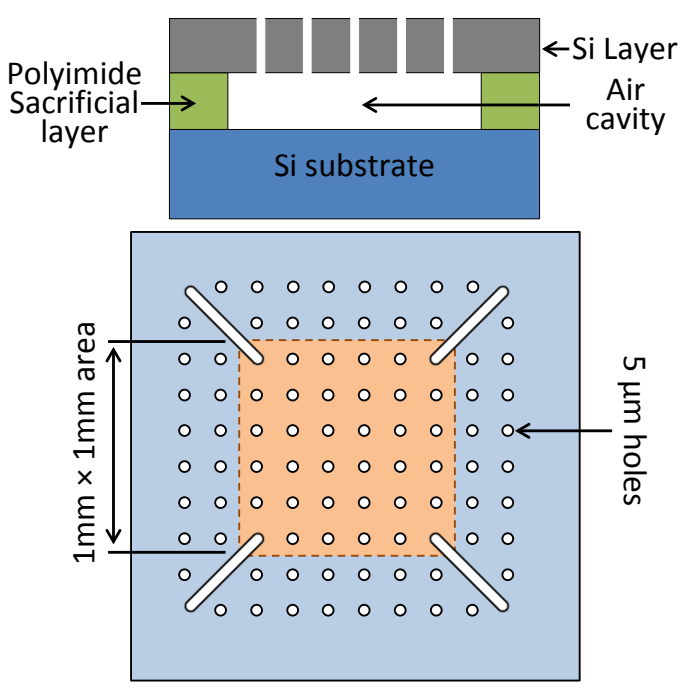

(a)

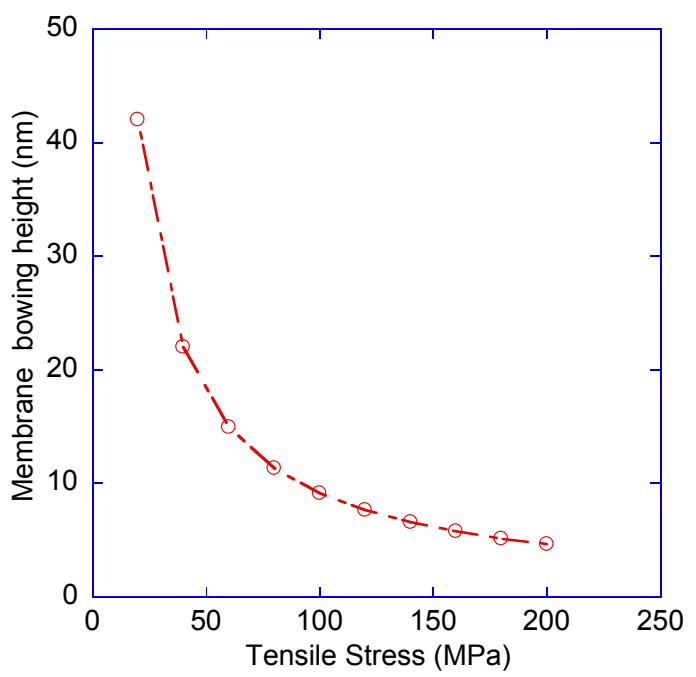

(b)

Fig. 4. FEM simulation of a $1 \mathrm{~mm}^{2}$ one-quarter wave thick suspended amorphous Si thin layer (thickness of $290 \mathrm{~nm}$ ): (a) structure cross section and top view (not to scale), and (b) membrane downward concave bowing as a function of Si residual tensile stress. Membrane bowing is defined as the maximum deviation in height of a layer from its ideally flat position (see Figure 3 (a)).

simulations were undertaken, using a nominal air cavity thickness of $1240 \mathrm{~nm}$ for the SWIR range and $2400 \mathrm{~nm}$ for the MWIR range, with the thickness of each layer as described in Table I. The suspended Si layers are assumed to be constrained at the centre of the optical area, remaining at the nominal thickness, and the edges of the Si layers move upwards at one edge and downwards at the other edge, producing a tilt variation measured from edge-to-edge of the $\mathrm{Si}$ layers as illustrated in Figure 6 (b). Simulated spectral transmittance as a function of wavelength of FP filters, using the proposed structure in Figure 6, for the SWIR and MWIR ranges are shown in Figure 7 (a) and (b), respectively. These simulations were undertaken for different values of tilt, maintaining the same average thickness value as the ideally flat and parallel filter, with the $\mathrm{Si}^{I}$ and $\mathrm{Si}^{I I}$ layers kept at a quarter wave optical thickness as depicted in Figure 6). These simulations indicate that an increase in tilt causes the peak transmittance of the filters to decrease and the FWHM to broaden. Figures 7 (c) and (d) illustrate the behaviour of peak transmittance and FWHM as a function of tilt for the SWIR and MWIR filters, respectively. It is noted that for both SWIR and MWIR filters a tilt that results in an edge-to-edge mirror displacement equal to the FWHM of the ideal filter (for these simulations $24 \mathrm{~nm}$ for SWIR filters and $48 \mathrm{~nm}$ for MWIR filters), reduces the peak transmittance to $64 \%$ and broadens the FWHM by $60 \%$. This modelling indicates that the fabrication process must be tightly 


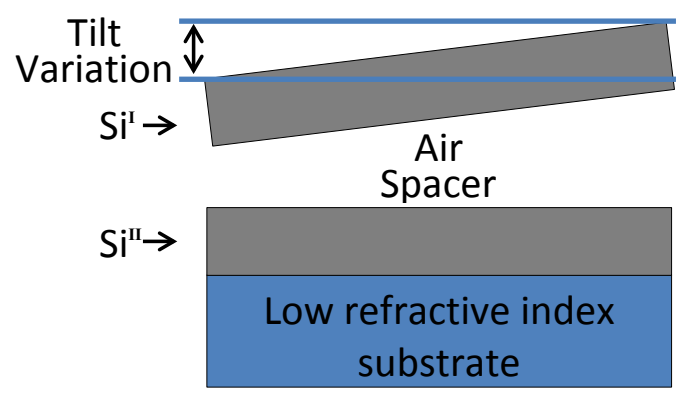

(a)

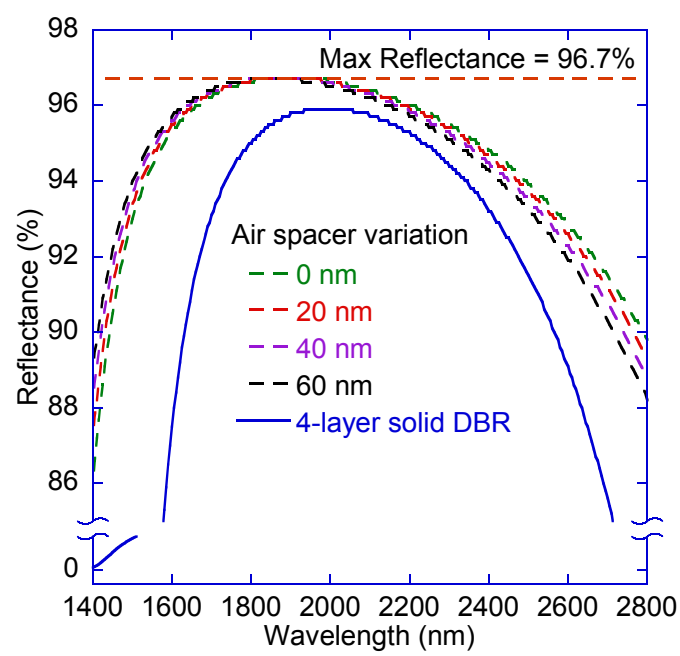

(c)

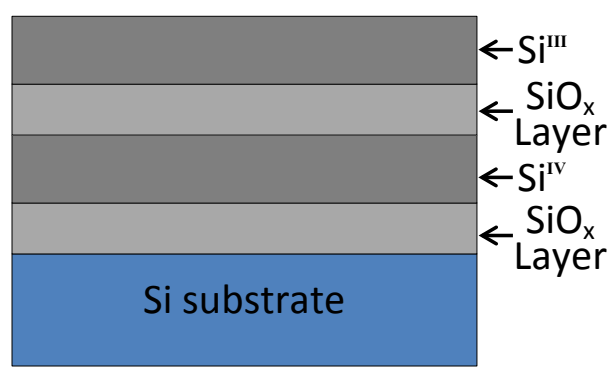

(b)

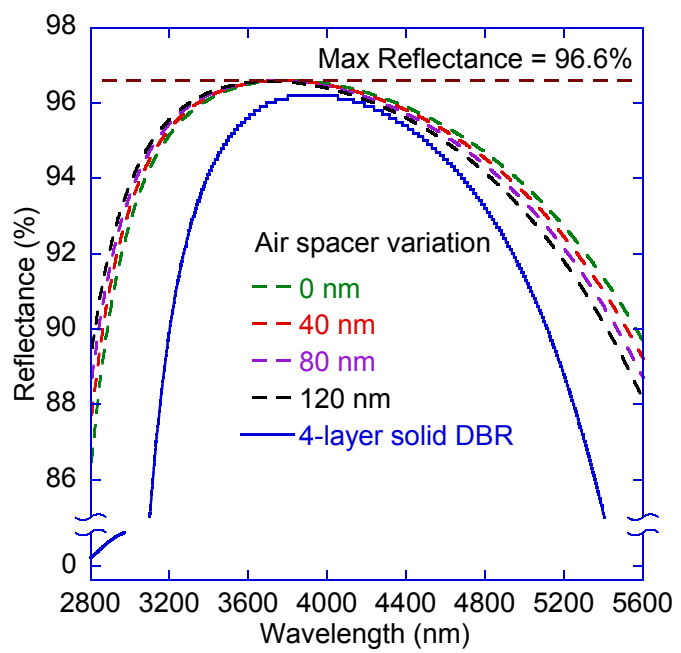

(d)

Fig. 5. Simulated reflectivity of $\mathrm{Si}$-air-Si DBRs as a function of downward tilt (bottom $\mathrm{Si}^{I I}$ layer flat relative to the substrate and the top suspended $\mathrm{Si}^{I}$ layer downward tilted relative to the substrate), and simulated reflectivity of ideal 4-layer solid DBRs: (a) Cross section of the simulated Si-air-Si DBR displaying downward tilt, (b) Cross section of the simulated ideal 4-layer DBR, (c) SWIR DBRs spectral reflectance and (d) MWIR DBRs spectral reflectance.

controlled to minimize the main cavity tilt below $20 \mathrm{~nm}$ for SWIR filters and $40 \mathrm{~nm}$ for MWIR filters in the suspended Si layers to produce high optical peak transmittance and narrow FWHM. That is the thickness variation across the surface of the sacrificial layer for both SWIR and MWIR filters needs to be less than $\sim 1.5 \%$ of the main air cavity nominal thickness.

\section{FABRICATION PROCESS}

The step-by-step fabrication process for the fixed cavity Fabry-Pérot filters is illustrated in Figure 8. Both the SWIR and MWIR filters employ an identical design; the difference being the thickness of the various material layers, as detailed in Table I. The fabrication process is as follows:

1) First, optical apertures were defined on a $300 \mu \mathrm{m}$ thick, double-side-polished silicon substrate using a lift-off process employing $10 \mathrm{~nm} \mathrm{Ti}$ and $100 \mathrm{~nm} \mathrm{Au}$ on the front side of the wafer (see Figure 8 (a)).

2) Subsequently, a Sentech 500D inductively coupled plasma enhanced chemical vapour deposition (ICPECVD) system was used to deposit a solid bottom DBR consisting of a silicon oxide/silicon/silicon oxide/silicon 4-layer stack, followed by a quarter-wave thick silicon oxide anti-reflection coating (ARC) on the back side of the substrate (see Figure 8 (b)).
3) Brewer Science Prolift 100-24 was spun on the wafer at $4200 \mathrm{rpm}$ for the MWIR filters and at $8000 \mathrm{rpm}$ for the SWIR filters to serve as the sacrificial layer for the main air cavity of the FP filters. The polyimide layer was soft-baked at $100{ }^{\circ} \mathrm{C}$ for 2 minutes and then hard-baked by ramping the temperature to $205{ }^{\circ} \mathrm{C}$ over a period of 15 minutes (see Figure 8 (c)). In order to fabricate filters with peak transmission at different wavelengths, an $\mathrm{O}_{2}$ plasma process was used to thin the sacrificial layer down to the desired thickness for each of the main air cavities of the SWIR and MWIR filters fabricated as specified in Table II.

4) A quarter-wave thick silicon layer was deposited on top of the sacrificial layer (see Table I), to serve as the bottom layer of the Si-air-Si DBR (see Figure 8 (d)).

5) Brewer Science Prolift 100-16 was spun on the wafer at $1400 \mathrm{rpm}$ for the MWIR filters and at $3000 \mathrm{rpm}$ for the SWIR filters to serve as the sacrificial layer for the DBR air spacer of the suspended DBR structure. The wafer was once again soft-baked at $100{ }^{\circ} \mathrm{C}$ for 2 minutes and then the temperature was ramped up to $205^{\circ} \mathrm{C}$ over a period of 15 minutes to cure the polyimide layer (see Figure 8 (e)).

6) The final quarter-wave thick silicon layer was deposited on top of the Prolift 100-16 to form the top layer of the 


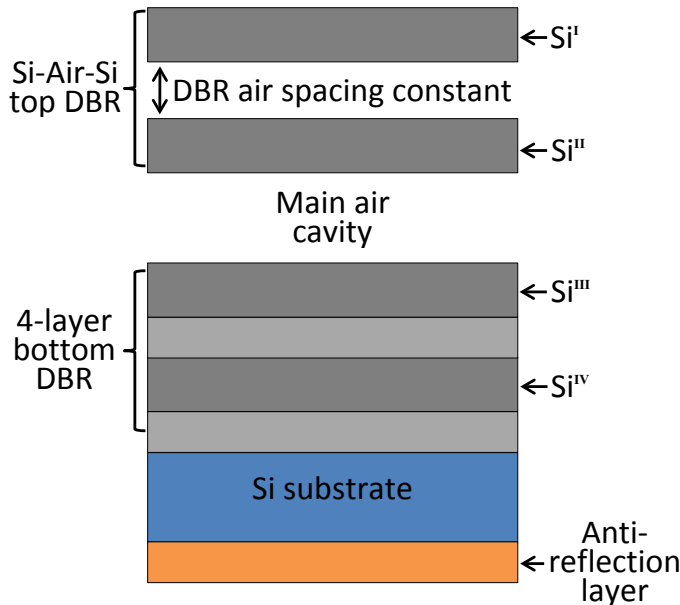

(a)

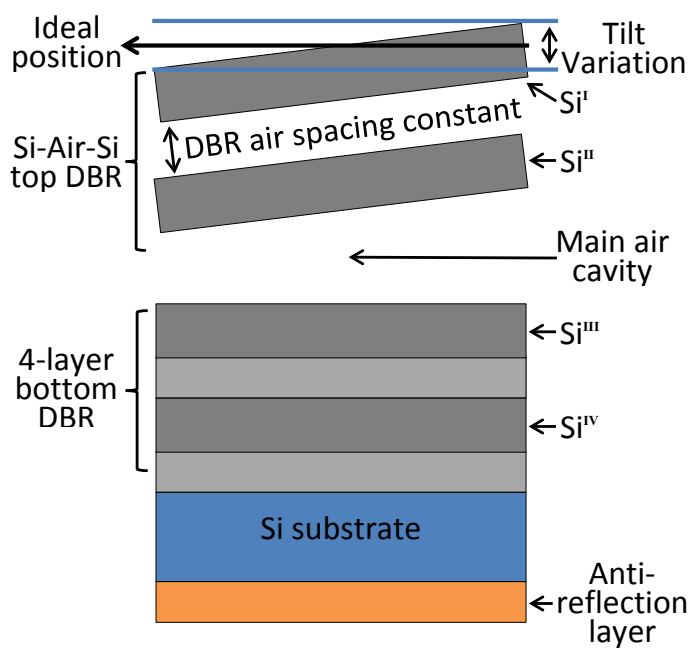

(b)

Fig. 6. Cross-section of the structure of the simulated filters: (a) Cross-section of the simulated ideal filters (b) Cross-section of the simulated filters displaying tilt in the top suspended DBR with constant separation for the DBR air spacer.

Si-air-Si DBR structure (see Figure 8 (f)).

7) A two-dimensional grid of circular etch holes, $5 \mu \mathrm{m}$ in diameter with a pitch of $60 \mu \mathrm{m}$, was etched through the top DBR layers down to the main optical cavity, along with four notches at the corners of the filter optical area. The etch holes, which facilitate release of the filter's resonant cavity, were formed using a 3 step dry-etch process. This involved a $\mathrm{CF}_{4}$ based etch of the top $\mathrm{Si}$ layer, followed by an $\mathrm{O}_{2}$ based plasmaetch to extend the holes through the Prolift 100-16 layer within the top DBR, and finally another $\mathrm{CF}_{4}$ based etch to create etch holes in the bottom silicon layer of the top DBR (see Figure $8(\mathrm{~g})$ ). The notches placed at the four corners of the top DBR are designed to minimize stress induced distortions at the corners of the optical area. Note that the etch holes do not have a significant effect on the spectral transmittance of the FP filters since their total area is approximately $1 \%$ of the optical area of the FP filter. An aligned two-dimensional grid of metal pads blocks this $1 \%$ component from entering the photodetector. These metal pads are formed at the same time as the metal optical shield that defines the filter optical area (see Figure 8 (a)).

8) As final step, both sacrificial layers were removed from the filter structure by etching in AZ 326 developer at room temperature, followed by a critical point drying process to yield two air cavities, as shown in Figure 8 (h).

\section{OPTICAL CHARACTERIZATION OF FP FILTERS}

The experimental component of this study included 8 SWIR and 8 MWIR FP filters, each with a different fixed main air cavity separating the two DBRs. The thickness of each layer used for the fabrication of the FP filters can be found in Table I, and the different values of each main air cavity length for each filter and peak wavelength position are shown in Table II for both the SWIR and MWIR ranges. The quality and performance of the fabricated fixed cavity FP filters was assessed using optical surface profilometry and optical spectral transmittance measurements.

\section{A. Optical surface profilometery}

Figures 9 (a) and (b) show the measured 3-D optical surface profiles of the fabricated $1 \mathrm{~mm} \times 1 \mathrm{~mm}$ filters designed for the SWIR and MWIR wavelength ranges, respectively. Corresponding surface profile line scans across the diagonal of the FP optical area are shown in Figures 9 (c) and (d). The regular variation present in the line scan of 9 (d) arises from proximity of the line scan to the regular etch-hole pattern. When referenced to the surface of the bottom mirror, the released SWIR and MWIR suspended filters show $15 \mathrm{~nm}$ and $20 \mathrm{~nm}$ of combined tilt and bowing in the suspended $\mathrm{Si}$ thin films, respectively. All the measured SWIR and MWIR filters displayed similar surface profiles in terms of surface topography.

\section{B. Spectral transmittance}

Filter spectral transmittance was measured using a Spectrum One FTIR spectrometer from Perkin Elmer. Measured and simulated data of the fabricated SWIR FP filters have been plotted in Figure 10 (a), in which the simulations were performed using the optical matrix method [16] extended to include the effect of measured 2-D tilt from the surface profile shown in Figure 9 (c) for each SWIR filter simulation. The peak transmittance wavelength, peak transmittance, FWHM and quality factor (Q-factor defined as the ratio of centre wavelength to the FWHM), for each SWIR filter presented in Figure 10 are listed in Table II. As shown in Figure 10 the measured optical spectral transmittance of the SWIR FP filters are in good agreement with the model results, since most of the transmittance and FWHM measurements are a close match with simulated values. For the SWIR filters fabricated in this work the measured peak transmittance ranges from 52-73\% 


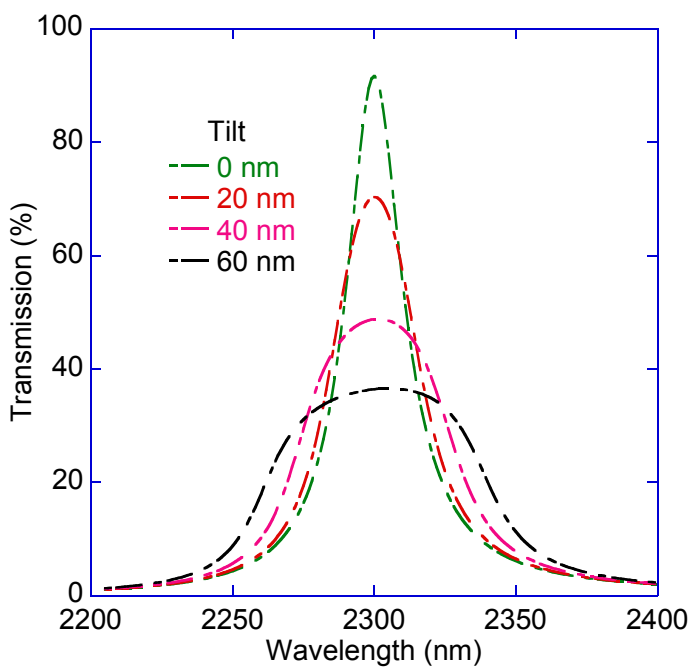

(a)

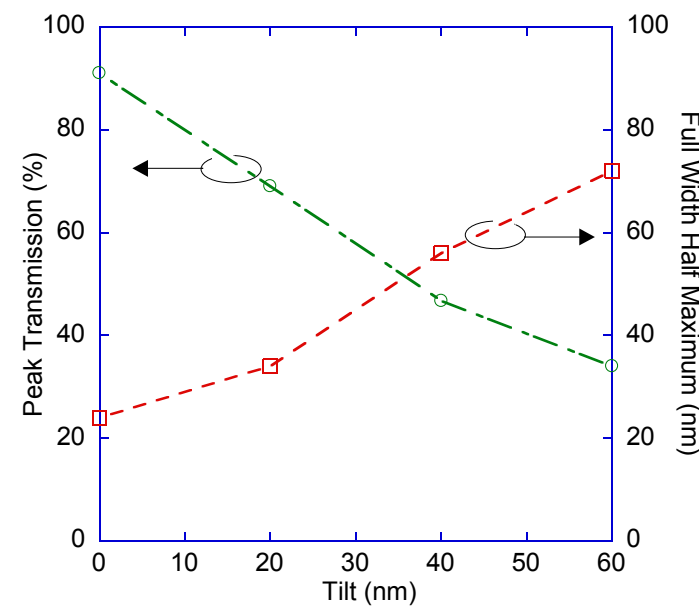

(c)

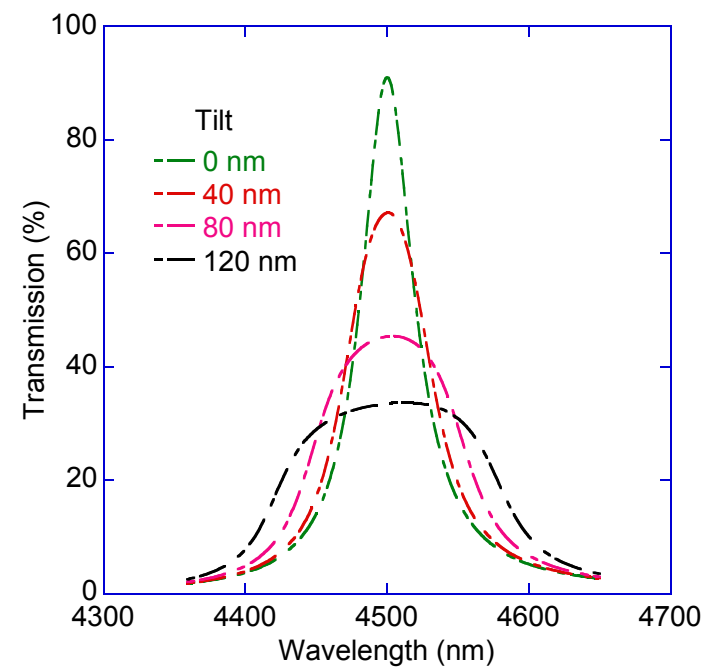

(b)

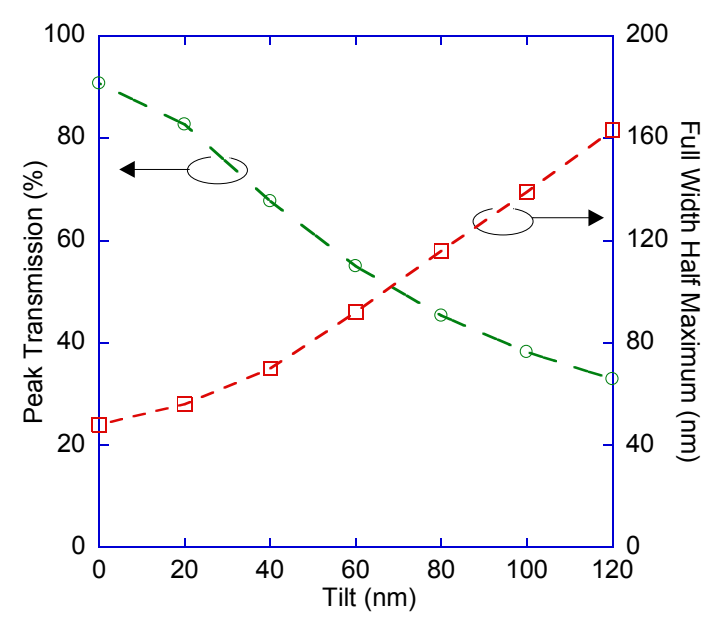

(d)

Fig. 7. Simulated spectral transmittance of FP filters using the structure proposed in Figure 6 for different values of tilt present in the $\mathrm{Si}^{I}$ and $\mathrm{Si}^{I I}$ layers (quarter wave optical thickness kept constant), leading to a linear variation in the main air cavity between the two DBRs (see Figure 6 (b)). Membrane tilt is defined as the maximum deviation in height of a layer from its ideally flat position, measured at opposite edges of the layer: (a) spectral transmittance of SWIR filter; (b) spectral transmittance of MWIR filter; (c) Peak transmission/FWHM vs Tilt for SWIR filter; (d) Peak transmission/FWHM vs Tilt for MWIR filter.

in excellent agreement with the $52-75 \%$ in the simulated data. The FWHM of the fabricated SWIR filters ranged from 27$60 \mathrm{~nm}$, as compared to simulated values of $29-49 \mathrm{~nm}$.

Measured and simulated data for the fabricated MWIR FP filters have been plotted in Figure 11 (a), in which the simulations were performed using the optical matrix method [16] extended to include the effect of measured 2-D tilt from the surface profile shown in Figure 9 (d) for each MWIR filter simulation. The peak transmittance position, peak transmittance, FWHM and quality factor (Q-factor) for each SWIR filter presented in Figure 11 are listed in Table II. As shown in Figure 11 the measured optical spectral transmittance of the SWIR FP filters are in good agreement with the modelled results, since most of the transmittance and FWHM measurements are a close match to the simulated values.

The measured peak transmittance of the fabricated MWIR filters was 6-15\% lower than the simulated peak transmittance, whereas the FWHM of the fabricated filters ranged from
$39 \mathrm{~nm}-113 \mathrm{~nm}$, in good agreement with simulation results. An interesting observation from Table II is that the MWIR filters have higher peak transmission and Q-factor compared to SWIR wavelength range filters, since the optical performance of a MWIR filter is less sensitive to a given thickness variation across the main air cavity. Note that the low measured peak transmittance value at the specific wavelength position of $4193 \mathrm{~nm}$ is likely a result of absorption due to atmospheric carbon-dioxide (see Table II and Figure 11 (a)), which can be corrected for by performing measurements in a vacuum or nitrogen environment. Current instrument limitations prevented this from being done. The high values of optical Q-factor of the SWIR and MWIR filters, which is an indicator of the spectral selectivity and quality of the filters is a result of the high degree of tilt and bowing control achieved during device fabrication. In all cases, experimental measurements yielded spectral transmittance in close agreement with simulated models. 


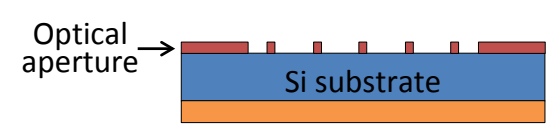

(a)

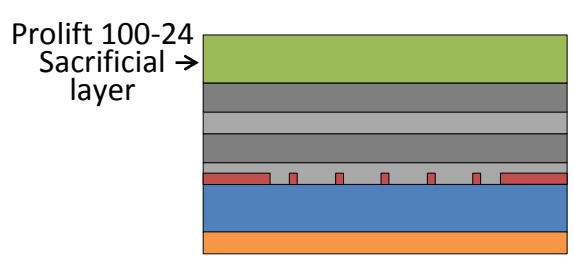

(c)

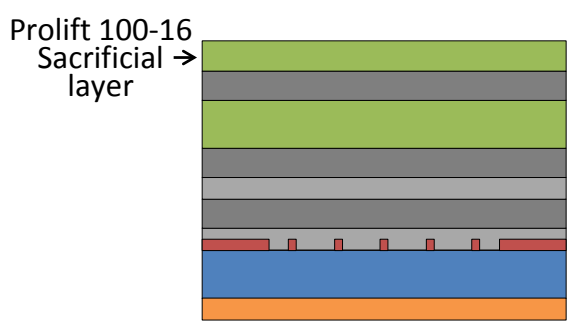

(e)

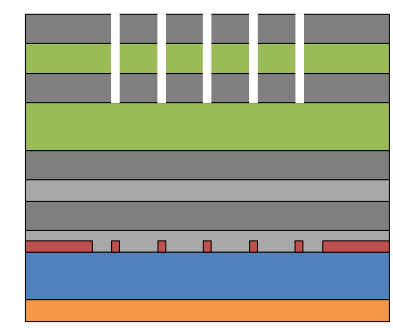

(g)

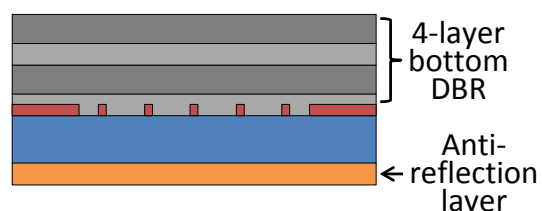

(b)

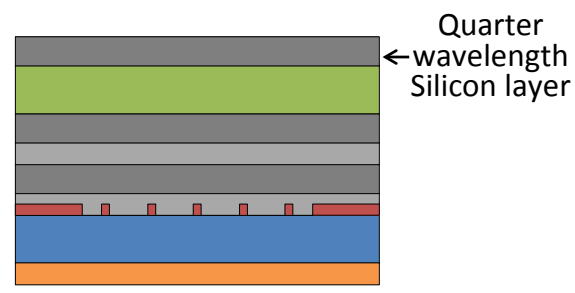

(d)

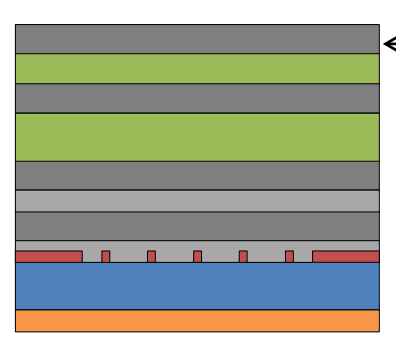

Quarter

$\leftarrow$ wavelength Silicon layer (f)

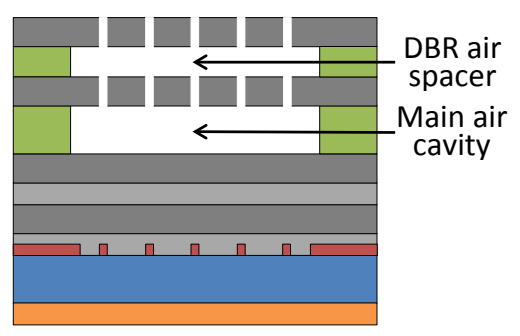

(h)

Fig. 8. Filter fabrication process: (a) deposition of metal aperture on silicon substrate to confine optical transmittance to the optical area of the FP filter; (b) deposition of silicon-silicon oxide based 4-layer bottom DBR and anti-reflection layers; (c) spinning, baking and patterning of Prolift 100-24 polyimide sacrificial layer for the main FP optical cavity; (d) deposition of quarter wavelength thick silicon thin film on top of Prolift 100-24; (e) spinning, baking and patterning of Prolift 100-16 sacrificial layer for the top DBR air spacer; (f) deposition of the quarter wavelength thick silicon thin film on top of Prolift 100-16; (g) patterning of etch holes through the top DBR layers; (h) wet release and critical point drying.

The spectral transmittance data in the SWIR and MWIR bands is plotted on a $\log$ scale in order to investigate the out-of-band rejection (ratio of out-of-band to pass-band transmittance) which can be achieved in these filters. As shown in Figure 10 (b), the typical experimental SWIR outof-band rejection is $30 \mathrm{~dB}$ compared to the simulated value of $50 \mathrm{~dB}$. However, the mid-wave data shown in Figure 11 (b) shows extremely good agreement between measured and modelled data, with $50 \mathrm{~dB}$ out-of-band rejection. This lower than expected SWIR out-of-band rejection is primarily a consequence of the lower signal-to-noise ratio performance of the FTIR spectrometer used for measurements in the SWIR range as compared to the MWIR range.

Table III shows a comparison of the peak transmittance and FWHM reported in this work with previously reported surface micromachined FP filters in the SWIR and MWIR wavelength ranges. It must be clarified that this work used fabricated fixed filters for proof of concept to estimate the tuning range and the expected performance, whereas all the other filters presented in Table III were fabricated as electrostatically-actuated filters. Table III also provides details of materials used for the top suspended DBRs of the filters, which clearly indicates that use of silicon dioxide and silicon monooxide as the low index DBR material resulted in a reported FWHM of 50-100 nm [13], [23] in the SWIR wavelength range and 57-200 nm [19], [24], [25], [12] in the MWIR wavelength range. The use of air as the low refractive index material in the top DBR has allowed this current work to attain the narrowest FWHM in the SWIR (27 nm) and MWIR (38 nm) wavelength ranges compared to all the previous works reported in Table III. Using the same number of layers for DBR formation (3layers) over the same wavelength range, the peak transmittance reported in earlier works ranged from 50\% - 80\% [13], [23], [19], [24], [25], [12], while filters reported in this 


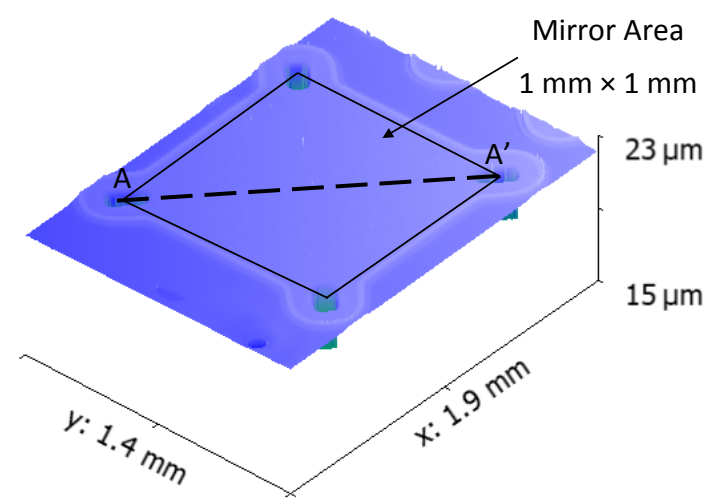

(a)

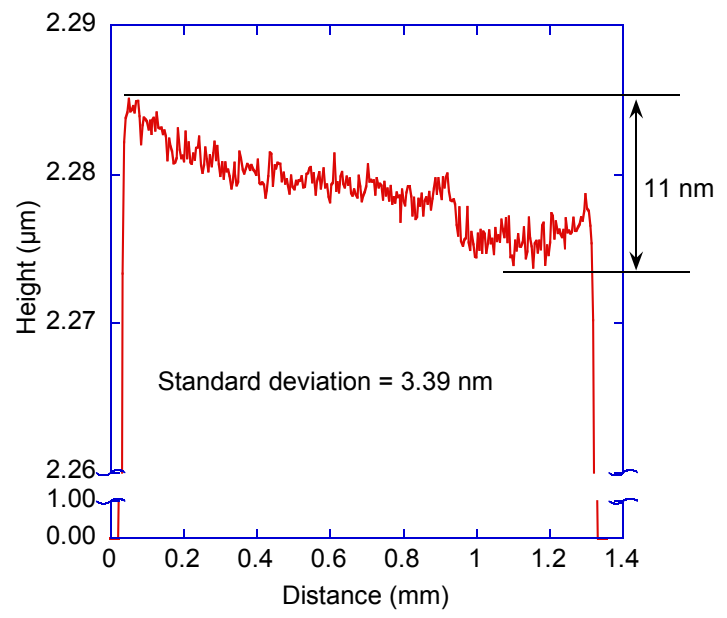

(c)

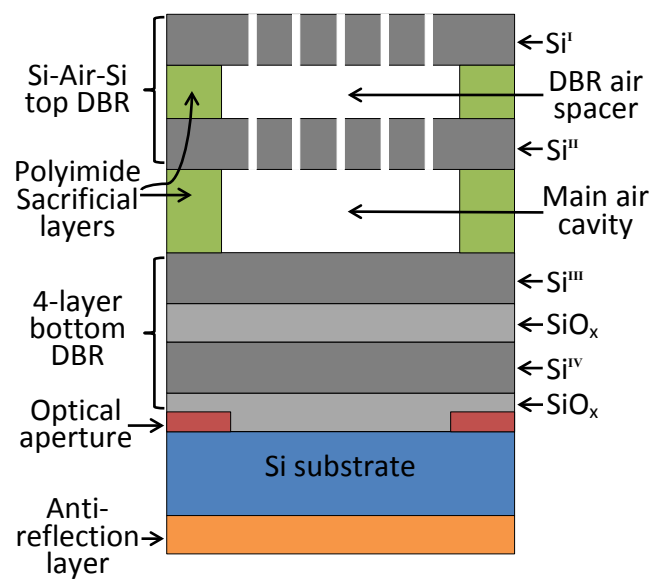

(b)

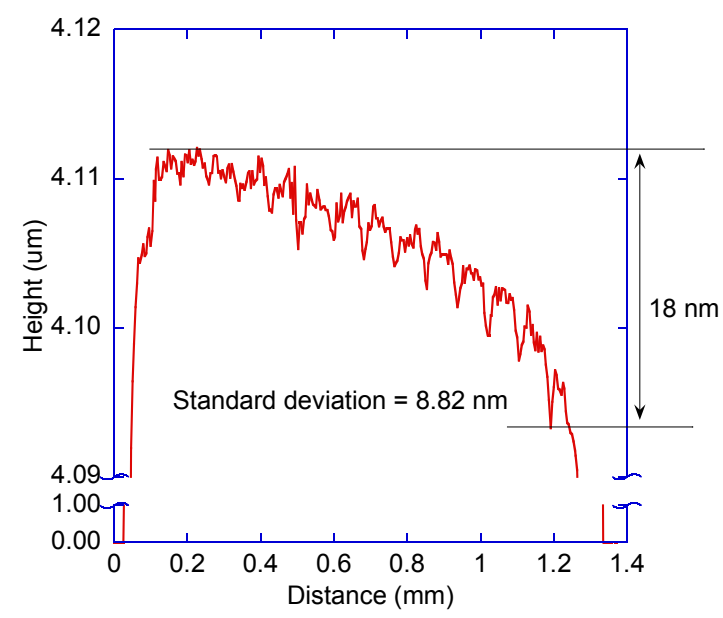

(d)

Fig. 9. Optical surface profile maps of $1 \mathrm{~mm} \times 1 \mathrm{~mm}$ filters fabricated (a) 3D surface plot of a SWIR filter, (b) cross-section view of the filter, (c) SWIR AA' and (d) MWIR AA' line scans across the diagonal of the optical FP area, between the notches of the filters, as shown in (a).

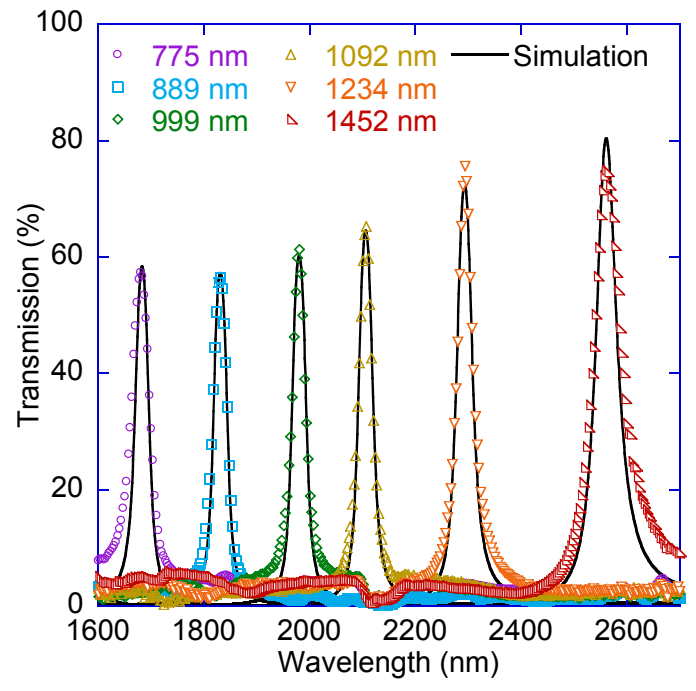

(a)

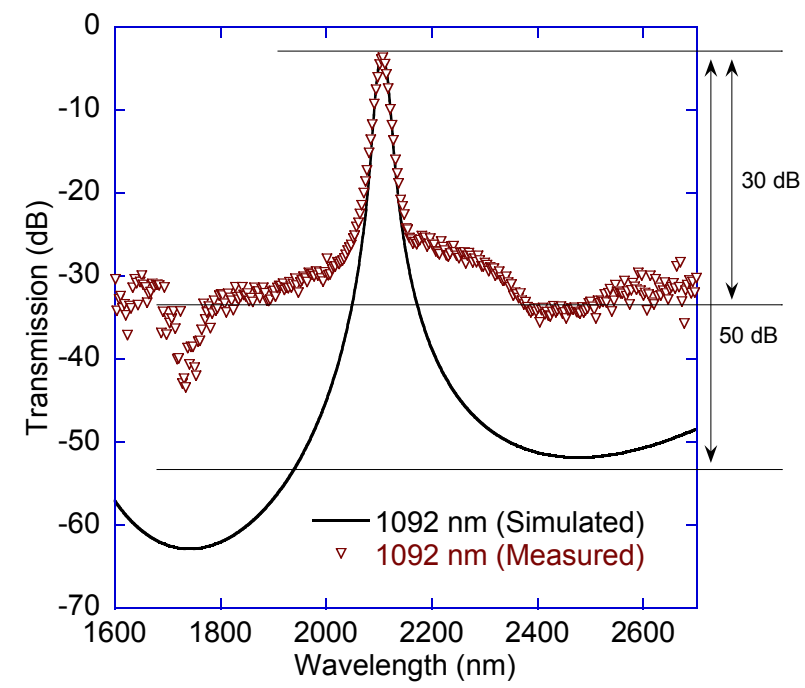

(b)

Fig. 10. (a) Measured and simulated spectral transmittance of SWIR FP filters. The nominal main air cavity of each filter is shown associated with each peak transmittance position. (b) Simulated and measured $1092 \mathrm{~nm}$ cavity filter plotted in dB, to examine extinction coefficient of the filters. 
TABLE II

SUMMARY OF MEASURED AND SIMULATED OPTICAL RESULTS OF FABRICATED AND MODELLED FP FILTERS.

\begin{tabular}{|c|c|c||c|c||c|c||c|c||}
\hline $\begin{array}{c}\text { WL } \\
\text { Range }\end{array}$ & $\begin{array}{c}\text { Center } \\
\text { WL } \\
\text { (nm) }\end{array}$ & $\begin{array}{c}\text { Main } \\
\text { cavity } \\
\text { (nm) }\end{array}$ & \multicolumn{2}{|c||}{ FWHM } & \multicolumn{2}{c|}{$\begin{array}{c}\text { Peak } \\
\text { Tx } \\
(\mathbf{\% m})\end{array}$} & \multicolumn{2}{|c|}{$\begin{array}{c}\text { Q } \\
\text { factor }\end{array}$} \\
\hline & & & Sim & Mes & Sim & Mes & Sim & Mes \\
\hline \hline & 1683 & 775 & 30 & 35 & 58 & 61 & 56 & 48 \\
& 1831 & 889 & 29 & 25 & 53 & 64 & 63 & 73 \\
& 1913 & 950 & 29 & 31 & 59 & 52 & 66 & 62 \\
SWIR & 1980 & 999 & 30 & 27 & 60 & 60 & 66 & 73 \\
& 2037 & 1041 & 30 & 27 & 62 & 63 & 68 & 75 \\
& 2106 & 1092 & 30 & 27 & 64 & 65 & 70 & 78 \\
& 2293 & 1234 & 33 & 33 & 73 & 74 & 69 & 69 \\
& 2561 & 1452 & 49 & 60 & 80 & 73 & 52 & 43 \\
\hline Mean & & & 32.5 & 33.1 & 63.6 & 64 & 63.9 & 65.2 \\
Std Dev & & & 6.8 & 11.4 & 8.8 & 7.1 & 6.5 & 13.2 \\
\hline \hline \multirow{5}{*}{} & 3361 & 1560 & 43 & 40 & 77 & 71 & 78 & 84 \\
& 3460 & 1637 & 41 & 38 & 76 & 73 & 84 & 91 \\
MWIR & 3605 & 1746 & 39 & 51 & 76 & 71 & 92 & 71 \\
& 3698 & 1816 & 39 & 44 & 77 & 74 & 95 & 84 \\
& 3792 & 1885 & 39 & 42 & 77 & 68 & 97 & 90 \\
& 4193 & 2180 & 43 & 87 & 81 & 48 & 97 & 48 \\
& 4380 & 2321 & 47 & 57 & 83 & 73 & 93 & 77 \\
& 4587 & 2480 & 54 & 70 & 84 & 70 & 85 & 66 \\
\hline Mean & & & 43.1 & 53.6 & 78.9 & 68.5 & 90.3 & 76.4 \\
Std Dev & & & 5.2 & 17.1 & 3.3 & 8.5 & 7 & 14.4 \\
\hline
\end{tabular}

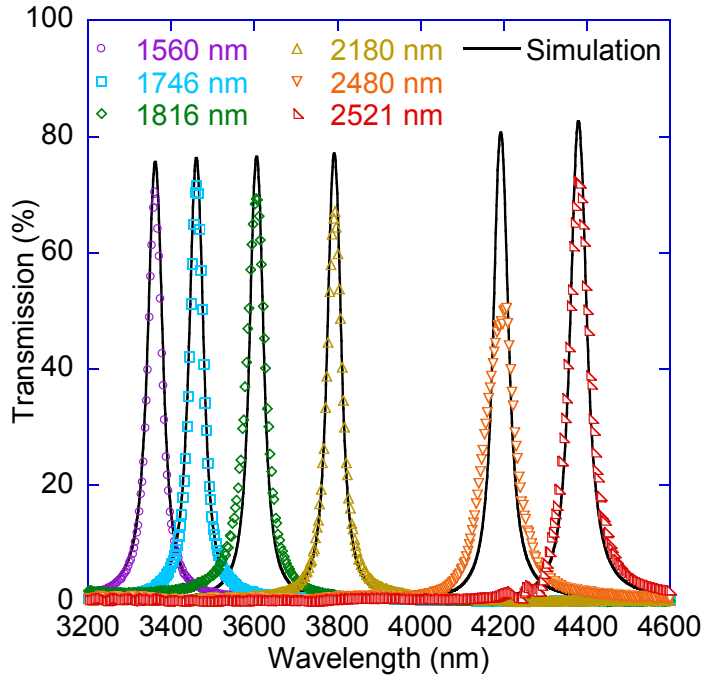

(a)

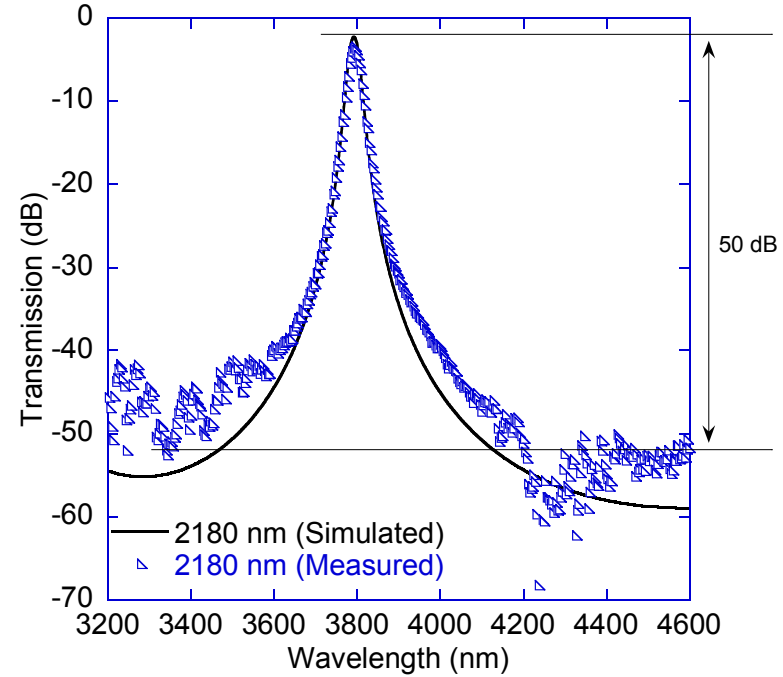

(b)

Fig. 11. (a) Measured and simulated spectral transmittance of MWIR FP filters. The nominal main air cavity is shown associated with each peak transmittance position and (b) simulated and measured $2080 \mathrm{~nm}$ cavity filter plotted logarithmically in dB units, to examine extinction coefficient of the filters.

paper have demonstrated comparable or better transmittance, with $61 \%-74 \%$ peak transmittance (excluding the peak positioned at $4193 \mathrm{~nm}$ due to carbon dioxide absorption). Our use of air as the low index material has resulted in narrow-band, high throughput FP filters with the possibility to achieve comparable or larger tuning ranges compared to previously reported studies. This approach based on a silicon-only MEMS platform supports a scalable process for high performance spectroscopic applications with challenging SWaP requirements.

\section{SUMmARY AND CONCLUSIONS}

This paper has presented the structural design and fabrication process for suspended Si-air-Si based DBR mirrors incorporated in fixed cavity Fabry-Pérot filters operating in the SWIR and MWIR wavelength ranges. The use of Si-airSi DBR suspended mirrors provides enhanced performance of the FP filters in comparison to other approaches used in previous studies due to the exceptionally large index contrast between silicon and air, and has simplified the stressbalancing requirements for the suspended DBR. The fabricated FP filters demonstrated a peak-to-peak variation in flatness of only $10-20 \mathrm{~nm}$ along the $1000 \mu \mathrm{m}$ dimension of the optical area. Spectral measurements yielded transmittance values in close agreement with simulated models. These large area $\left(1 \mathrm{~mm}^{2}\right)$ filters represent promising candidates for spectroscopic sensing and imaging in the SWIR and MWIR 
TABLE III

COMPARISON OF THE RESULTS PRESENTED IN THIS WORK WITH EARLIER REPORTED STUDIES ON SURFACE MICROMACHINED MEMS BASED FP FILTERS.

\begin{tabular}{|c||c||c||c||c||c||c|}
\hline $\begin{array}{c}\text { WL } \\
\text { Range }\end{array}$ & Reference & $\begin{array}{c}\text { Top DBR } \\
\text { Material }\end{array}$ & $\begin{array}{c}\text { Center } \\
\text { WL } \\
(\mathbf{n m})\end{array}$ & FWHM & $\begin{array}{c}\text { Peak } \\
\text { Tx }\end{array}$ & $\begin{array}{c}\text { Tuning } \\
\text { Range } \\
\text { nm }\end{array}$ \\
\hline \hline \multirow{2}{*}{ SWIR } & Milne et al. [13] & $\mathrm{Ge} / \mathrm{SiO} / \mathrm{Ge}$ & 2100 & 50 & $50 \%$ & $1615-2425$ \\
& Musca et al. $[23]$ & $\mathrm{Ge} / \mathrm{SiO} / \mathrm{Ge}$ & 1950 & 100 & $60 \%$ & $1850-2200$ \\
& This work & $\mathrm{Si} / \mathrm{air} / \mathrm{Si}$ & 2106 & 27 & $65 \%$ & - \\
\hline \multirow{5}{*}{ MWIR } & Milne et al. $[19]$ & $\mathrm{Ge} / \mathrm{SiO} / \mathrm{Ge}$ & 3900 & 200 & $50 \%$ & $3250-4320$ \\
& Meinig et al. $[24]$ & $\mathrm{Si} / \mathrm{SiO} / \mathrm{Si}$ & 4100 & 57 & $80 \%$ & $4050-4950$ \\
& Ebermann et al. $[25]$ & $\mathrm{Si} / \mathrm{SiO} / \mathrm{Si}$ & 3400 & 80 & $80 \%$ & $3020-4320$ \\
& Quack et al. $[12]$ & Lead $\mathrm{Chalcogenide/EuTe}$ & 4850 & 100 & - & $4700-5400$ \\
& This work & Si/air/Si & 3460 & 38 & $73 \%$ & - \\
\hline
\end{tabular}

wavelength range, specifically in applications that demand very low size, weight and power.

\section{ACKNOWLEDGMENT}

This work was performed in part at the Western Australian node of the Australian National Fabrication Facility (ANFF). This research was supported financially by the Grains Research and Development Corporation, as well as by a number Australian Research Council research grants (DP 0881579, DP 140101766). We also acknowledge support from the Western Australian State Government Office of Science and the Centre for Microscopy, Characterisation and Analysis at UWA.

\section{REFERENCES}

[1] R. Linares, G. Vergara, R. Gutierrez, C. Fernandez, V. Villamayor, L. Gomez, M. Gonzalez-Camino, and A. Baldasano, "Gas and flame detection and identification using uncooled mwir imaging sensors," in SPIE Sensing Technology+ Applications. International Society for Optics and Photonics, 2015, pp. 94 851F-94 851F.

[2] R. S. Allison, J. M. Johnston, G. Craig, and S. Jennings, "Airborne optical and thermal remote sensing for wildfire detection and monitoring," Sensors, vol. 16, no. 8, p. 1310, 2016.

[3] V. Adamchuk, J. Hummel, M. Morgan, and S. Upadhyaya, "On-the-go soil sensors for precision agriculture," Computers and Electronics in Agriculture, vol. 44, no. 1, pp. 71 - 91, 2004. [Online]. Available: http://www.sciencedirect.com/science/article/pii/S0168169904000444

[4] R. Gebbers and V. I. Adamchuk, "Precision agriculture and food security," Science, vol. 327, no. 5967, pp. 828-831, 2010. [Online]. Available: http://science.sciencemag.org/content/327/5967/828

[5] W. S. Lee and R. Ehsani, "Sensing systems for precision agriculture in florida," Computers and Electronics in Agriculture, vol. 112, no. Supplement C, pp. 2 - 9, 2015, precision Agriculture. [Online]. Available: http://www.sciencedirect.com/science/article/pii/S0168169914002865

[6] R. V. Rossel, D. Walvoort, A. McBratney, L. Janik, and J. Skjemstad, "Visible, near infrared, mid infrared or combined diffuse reflectance spectroscopy for simultaneous assessment of various soil properties," Geoderma, vol. 131, no. 1, pp. 59 - 75, 2006. [Online]. Available: http://www.sciencedirect.com/science/article/pii/S0016706105000728

[7] R. Goodacre, "Explanatory analysis of spectroscopic data using machine learning of simple, interpretable rules," Vibrational Spectroscopy, vol. 32, no. 3, pp. 33-45, 2003.

[8] H. Saari, I. Pellikka, L. Pesonen, S. Tuominen, J. Heikkilãd', C. Holmlund, J. MÃd'kynen, K. Ojala, and T. Antila, "Unmanned aerial vehicle (UAV) operated spectral camera system for forest and agriculture applications," C. M. U. Neale and A. Maltese, Eds., p. $81740 \mathrm{H}$.

[9] H. Saari, A. AkujÃd'rvi, C. Holmlund, H. Ojanen, J. Kaivosoja, A. Nissinen, and O. NiemelÃd'inen, "Visible, very near IR and short wave IR hyperspectral drone imaging system for agriculture and natural water applicationS," vol. 42, pp. 165-170.
[10] B. Saadany, M. Malak, F. Marty, Y. Mita, D. Khalil, and T. Bourouina, "Electrostatically-tuned optical filter based on silicon bragg reflectors," in IEEE/LEOS international conference on optical MEMS and their applications, 2006, pp. 86-87.

[11] Y. Hongbin, Z. Guangya, C. F. Siong, L. Feiwen, W. Shouhua, and Z. Mingsheng, "An electromagnetically driven lamellar grating based fourier transform microspectrometer," Journal of Micromechanics and Microengineering, vol. 18, no. 5, pp. 1-6, 2008.

[12] N. Quack, S. Blunier, J. Dual, F. Felder, M. Arnold, , and H. Zogg, "Midinfrared tunable resonant cavity enhanced detectors," Sensors, vol. 8 , no. 9, pp. 5466-5478, 2008.

[13] J. S. Milne, J. M. Dell, A. J. Keating, and L. Faraone, "Widely tunable MEMS-based FabryâĂŞPerot filter," Journal of Microelectromechanical Systems, vol. 18, no. 4, pp. 905-913, 2009.

[14] R. St-Gelais, A. Poulin, and Y. A. Peter, "Advances in modeling, design, and fabrication of deep-etched multilayer resonators," Journal of Lightwave Technology, vol. 30, no. 12, pp. 1900-1908, 2012.

[15] D. K. Tripathi, H. Mao, K. K. M. B. D. Silva, J. W. Bumgarner, M. Martyniuk, J. M. Dell, and L. Faraone, "Large-area mems-based distributed bragg reflectors for short-wave and mid-wave infrared hyperspectral imaging applications," Journal of Microelectromechanical Systems, vol. 24, no. 6, pp. 2136-2144, 2015.

[16] H. A. Macleod, Thin-Film Optical Filters. Institute of Physics, London, 2001.

[17] G. Hernandez, Fabry-Perot Interferometers. Cambridge University Press,London, 1986.

[18] J. M. Vaughan, The Fabry-Perot Interferometer History,Theory,Practice and Applications. Taylor and Francis, New York, 1989.

[19] J. S. Milne, "Micro-electromechanical technologies for next-generation spectroscopic systems," Ph.D. dissertation, School of Electrical, Electronics and Computer Engineering, 2009.

[20] M. Martyniuk, J. Antoszewski, C. Musca, J. Dell, and L. Faraone, "'stress in low-temperature plasma enhanced chemical vapour deposited silicon nitride thin films," Smart Materials and Structures, vol. 15, no. 1, pp. S29-S38, 2006.

[21] CoventorWare - MEMS design and simulation software suite I coventor. [Online]. Available: https://www.coventor.com/memssolutions/products/coventorware/

[22] R. Kuschnereit, H. Fath, A. Kolomenskii, M. Szabadi, and P. Hess, "Mechanical and elastic properties of amorphous hydrogenated silicon films studied by broadband surface acoustic wave spectroscopy," Applied Physics A, vol. 61, no. 3, pp. 269,276, 1995.

[23] C. A. Musca, J. Antoszewski, K. J.Winchester, A. J. Keating, T. Nguyen, K. K. M. B. D. Silva, J. M. Dell, L. Faraone, P. Mitra, J. D. Beck, M. R. Skokan, and J. E. Robinson, "Monolithic integration of an infrared photon detector with a mems-based tunable filter," IEEE Electron Device Letters, vol. 26, no. 12, pp. 888-890, 2005.

[24] M. Meinig, M., N. Neumann, S. Kurth, K. Hiller, and T. Gessner, "Dual-band MEMS FABRY-PEROT filter with two movable reflectors for mid- and long-wave infrared microspectrometers," in 16th International Solid-State Sensors, Actuators and Microsystems Conference (TRANSDUCERS), 2011, pp. 2538 - 2541.

[25] M. Ebermann, K. Hiller, S. Kurth, and N. Neumann, "Design, operation and performance of a Fabry-Perot-Based MWIR microspectrometer," in Proceedings-OPTO 2009 and IRS 2009, 2009, pp. 233-238. 